\title{
Almond snack consumption improves endothelial function in adults with moderate risk of cardiovascular disease: a randomised, controlled, parallel trial
}

\author{
Vita Dikariyanto, Sarah Berry, Leanne Smith, Lucy Francis, May Robertson, Eslem Kusaslan, \\ Molly O'Callaghan-Latham, Peter Ellis, Phil Chowienczyk and Wendy Hall \\ King's College London, London, United Kingdom
}

\section{Abstract}

Endothelial dysfunction is a predictor for cardiovascular disease risk and is a key feature of atherosclerosis. Poor diet quality, including consumption of saturated fat-rich, high-refined carbohydrate snack foods, may have adverse effects on endothelial function. Thus, snack foods, which contribute an average of $20 \%$ of energy intake in the UK adult population, present an easily identifiable target to improve vascular health. Almonds are nutrient-dense foods that are rich in unsaturated fats, fibre, minerals and non-nutrient bioactives (NNB), and may have health benefits by displacing snacks high in refined carbohydrates, enriching the diet with micronutrients and NNB, and/or low lipid bioaccessibility. Human clinical trials have demonstrated LDL cholesterol-lowering effects of daily almond consumption, yet the effects on endothelial function are unclear. This study aimed to investigate whether replacing habitual snacks ( $20 \%$ estimated daily energy requirements) with almonds had any impact on endothelium-dependent vasodilation, measured by flow-mediated dilatation (FMD) using ultrasound imaging of the brachial artery following reactive hyperaemia. A randomised, controlled, parallel trial in adult regular snack consumers aged 30-70 y at moderate risk of cardiovascular disease was conducted, including a 2-week run-in period with control snacks and a 6-week intervention period. Control sweet and savoury mini muffin snacks were developed to replicate the average UK snack nutrient profile, which was calculated from snack foods identified in the UK National Diet and Nutrition Survey (NDNS) database (55\% energy from carbohydrate, 36\% total fat (14\% saturated fat), and 10\% protein). One hundred and nine volunteers ( 77 females and 32 males; mean age 56 y) were enrolled in the study and 107 were randomised to isocaloric treatments, 1) control muffins, or 2) dry roasted whole almonds; 105 participants completed the study. Almonds significantly increased FMD relative to control (mean difference $3.6 \%, 95 \%$ CI $1.7,5.5 ; P<0.001$ ), indicating improved endothelial function, and LDL-cholesterol (mean difference $-0.25 \mathrm{mmol} / \mathrm{L}, 95 \% \mathrm{CI}-0.47,-0.03 ; P=0.030$ ) significantly decreased adjusted with sex, age and baseline BMI and baseline dependent outcome values. Snacking on whole almonds as a replacement for snacks high in refined starch and sugar, and low in fibre and unsaturated fatty acids, improves endothelial function. The results of this study provide further evidence for the importance of nuts in dietary strategies to reduce risk of cardiovascular disease.

\section{Conflict of Interest}

There is no conflict of interest. 